\title{
PIWI-like protein I upregulation promotes gastric cancer invasion and metastasis
}

\author{
Chun-li Gao ${ }^{1, *}$ \\ Rui Sun ${ }^{2, *}$ \\ Dong-hai $\mathrm{Li}^{3}$ \\ Fei Gong ' \\ 'Department of Gastroenterology, \\ 2Department of Oncology, \\ ${ }^{3}$ Department of Pathology, The First \\ People's Hospital of Lanzhou City, \\ Lanzhou, Gansu, 730050, China \\ *These authors contributed equally \\ to this work
}

This article was published in the following Dove Press journal: OncoTargets and Therapy

Background: PIWI-like protein 1 (PIWIL1) is an important member of the Argonaute protein family and is closely related to the malignant behaviors of tumor cells. This study aimed to investigate the relationship between PIWIL1 and gastric cancer (GC).

Methods: We investigated PIWIL1 expression status in GC tissues as well as its association with clinicopathological characteristics and prognosis of GC patients. PIWIL1 siRNA was transfected into a GC cell line to elucidate its impact on malignant biological behavior.

Results: The results showed that PIWIL1 was upregulated in GC tissues and correlated with tumor differentiation, lymph node status, and TNM stage. The high PIWIL1 expression was an independent predictor for the prognosis of patients with GC. Silencing of PIWIL1 expression in GC cell lines suppressed tumor cell proliferation, migration, and invasion.

Conclusion: High PIWIL1 expression suggests a poor prognosis for GC patients and PIWIL1 can serve as an important molecular marker for predicting the prognosis of GC patients.

Keywords: gastric cancer, PIWIL1, invasion, metastasis

\section{Introduction}

Gastric cancer (GC) is one of the most common malignant tumors, and is associated with poor therapeutic effect and dismal prognosis. Globally, the morbidity and mortality of GC rank fifth and third, respectively, among malignant tumors. ${ }^{1}$ The diagnostic techniques and therapeutic methods for GC are increasingly improving, and the overall survival (OS) of GC patients has also been enhanced. However, some patients develop disease recurrence soon after surgery and experience distant metastasis, ${ }^{2}$ which is the leading cause of death among GC patients. Consequently, exploring the molecular mechanisms behind gastric carcinogenesis and metastasis has become an important area of research.

Tumors derive from a small fraction of stem cells, which have highly proliferative, continuous self-renewal, and multi-lineage differentiation capacities. ${ }^{3}$ These capacities of stem cells are closely correlated with the PIWI-like proteins. ${ }^{4}$ The PIWI-like protein 1 (PIWIL1) is an important member of the Argonaute protein family and plays a vital role in stem cell proliferation, embryogenesis, growth, development, differentiation, and maturation in multiple organisms. ${ }^{4-6}$ In addition, PIWIL1 is closely related to the malignant behaviors of tumor cells. Previous studies have found that PIWIL1 is highly expressed in colorectal cancer tissues, and its expression is related to the lymph node status and disease stage of patients. ${ }^{7}$ It has been found to be a dismal prognostic factor for patients with colorectal cancer. Nevertheless, research on the relationship between PIWIL1 and GC is rare. 
In the current study, we examined PIWIL1 expression in GC and analyzed its relationship with the clinicopathological features of GC patients. Furthermore, the relationship between PIWIL1 and the malignant phenotype of GC cells was evaluated.

\section{Methods}

\section{Patients and cell line}

We collected 120 GC cases and corresponding para-carcinoma tissues surgically resected in The First People's Hospital of Lanzhou City from January 2008 to December 2014. All patients were diagnosed pathologically and had complete clinical data. The cohort included 83 men and 37 women, with an age range of 32-74 years (average, $47.1 \pm 8.4$ years). Patients were clinically classified using the TNM classification system that was formulated jointly by the American Joint Committee on Cancer/Union for International Cancer Control. ${ }^{8}$ All patients were naive to chemotherapy, targeted therapy, or biotherapy before surgery. All patients provided written informed consent for study participation. All of the procedures were approved by the Ethics Committee of The First People's Hospital of Lanzhou City, and were conducted in accordance with the principles of the Declaration of Helsinki.

The human GC cell lines NCI-N87, AGS, and MGC803 and the normal gastric epithelial cell line GES-1 were purchased from the Type Culture Collection of the Chinese Academy of Sciences (Shanghai, China) and cultured in DMEM containing $10 \%$ FBS. The medium was replaced every 3 days.

\section{Immunohistochemical analysis}

The paraffin-embedded specimens were sliced continuously, followed by conventional deparaffinization and hydration using xylenes and ethanol. The sections were then placed into the citrate antigen-retrieval solution for antigen retrieval, followed by washing with PBS twice. The sections were placed into $1.5 \% \mathrm{H}_{2} \mathrm{O}_{2}$ in deionized water for 30 minutes and then washed with distilled water. Subsequently, the sections were incubated in normal serum for 15 minutes at $37^{\circ} \mathrm{C}$, followed by incubation with primary antibody (rabbit anti-human PIWIL1 antibody, 1:500; Abcam, Cambridge, UK; PBS was used instead of the primary antibody as a negative control) at $4^{\circ} \mathrm{C}$ overnight. Secondary antibody was added after immersion in PBS and the slides were incubated at $37^{\circ} \mathrm{C}$ for 60 minutes, followed by $3^{\prime}$-diaminobenzidine developing for 30 minutes, the addition of peroxidase-labeled streptomycin at $37^{\circ} \mathrm{C}$ for 20 minutes, and hematoxylin counterstaining and sealing.
Five medium fields of view $(\times 200)$ were randomly selected, from which 200 tumor cells in each field of view were counted (a total of 1,000 cells were counted). The staining intensity was defined as follows: 0 , no staining; 1 , faint yellow staining; 2, claybank staining; and 3, brown staining. The proportion of cells with positivity was rated from $0 \%$ to $100 \%$. In the current study, a product of staining intensity and the proportion of positive cells $>40 \%$ was defined as high PIWIL1 expression; otherwise, it was defined as low expression.

\section{Cell transfection}

MGC803 cells were transfected using the Lipofectamine 2000 kit (Invitrogen; Thermo Fisher Scientific, Waltham, MA, USA) in six-well plates at a density of $5.0 \times 10^{5}$ cells/well for 24 hours. PIWIL1 expression was downregulated using small interfering RNA (siRNA), with the sequence 5'-AGTCA GCAACCTGGTTATA-3'. MGC803 cells that were not subjected to any treatment was used as the negative control. Cells were incubated at $37^{\circ} \mathrm{C}$ in $5 \% \mathrm{CO}_{2}$ for 72 hours and were then collected for subsequent experiments.

\section{Western blotting}

Protein was extracted from tissues or cells using protein lysis buffer, and protein concentration was measured using the bicinchoninic acid disodium kit (Beyotime, Shanghai, China). Equivalent amounts of cell lysate were electrophoresed on a $10 \%$ SDS-PAGE gel and transferred onto polyvinylidene fluoride membranes. The membranes were sealed and incubated with primary antibody (rabbit antihuman PIWIL1 antibody, 1:500; Abcam, Cambridge, UK; PBS was used instead of the primary antibody as a negative control). GAPDH (Multisciences, Shanghai, China) was used as the internal reference. Subsequently, the membranes were incubated with secondary antibody goat anti-rabbit immunoglobulin G (IgG, 1:1,000; Multisciences). The band intensity was analyzed using Image-Pro Plus software (Version 6.0, Media Cybernetics, Inc., Rockville, MD, USA).

\section{Cell proliferation assay}

Cell proliferation was measured using the Cell Counting Kit-8 (CCK-8; Dojindo, Kumamoto, Japan). In brief, cells were plated in 96-well plates at a density of $3 \times 10^{3}$ cells/well, and $10 \mu \mathrm{L}$ CCK-8 reagent was added to each well after $24,48,72$, and 96 hours. Finally, the optical density was measured using a cell analyzer (Bio-Rad Laboratories Inc., Hercules, CA, USA) at $450 \mathrm{~nm}$. Each experiment was repeated three times. 


\section{Transwell assay}

Cell invasion was determined using Matrigel Transwell chambers (BD Biosciences, Franklin Lakes, NJ, USA) that were used to evaluate the invasion capacity. The lower chamber was filled with medium supplemented with $20 \%$ FBS as the chemotactic agent. After 24-hour incubation, the invading cells were fixed on the membranes, stained with crystal violet, and counted. For the migration test, all operations were consistent with the invasion test, except that the six-well chamber was without Matrigel.

\section{Wound-healing assay}

The cell-migration capacity was further verified using a scratch assay. In brief, cells were inoculated into six-well plates to grow until confluence. Subsequently, the singlelayer cells were scratched using a $200-\mu \mathrm{L}$ pipette tip and washed with PBS twice. The scratch regions were imaged at 0 and 24 hours using a microscope.

\section{Statistical analysis}

The two groups were compared using Student's $t$-test. The chi-squared test were used to determine the statistical significance of differences between the groups. The main outcome measure was disease-free survival (DFS) and OS. Cumulative patient survival was estimated using the Kaplan-Meier method, and a log-rank test was used to compare the survival curves. A Cox proportional hazards model was used to calculate univariate and multivariate HRs for the variables. Values of $P<0.05$ were considered statistically significant. All statistical analyses were carried out using the SPSS 18.0 statistical software package (SPSS Inc., Chicago, IL, USA).

\section{Results}

\section{PIWILI expression in GC}

In accordance with results of immunohistochemical staining, the PIWIL1 protein is mainly located in the cytoplasm of the GC cells (Figure 1A) and a lot of this protein also expressed in the nucleus (Figure 1B). According to the immunohistochemical evaluation criteria, the $120 \mathrm{GC}$ patients were stratified into a high PIWIL1 expression group and a low PIWIL1 expression group. Moreover, we analyzed the relationship between PIWIL1 expression status and the clinicopathological features of GC. Our results demonstrated that PIWIL1 expression status was closely correlated with tumor differentiation degree, depth of invasion, lymph node status, and TNM stage, but not sex, age, or pathological subtype. The results are summarized in Table 1.

\section{PIWILI and prognosis of GC patients}

We investigated the relationship between the expression of PIWIL1 and prognosis of GC patients by long-term follow-up. The survival curve was derived from the data. The Kaplan-Meier survival model suggested that the survival time, whether DFS or OS, of GC patients in the high PIWIL1 expression group was notably lower than in the low PIWIL1 expression group (Figure 2A and B). As with other studies, patients with advanced GC had worse survival than those with early GC (Figure 2C). In addition, we found that GC patients with Helicobacter pylori infection had shorter survival (Figure 2D).

All variables affecting the OS of GC patients were analyzed using the Cox proportional hazard model. Results of single-factor analysis suggested that the prognosis of GC patients was closely related to PIWIL1 expression. Moreover, lymph node metastasis, TNM stage, H. pylori infection, and PIWIL1 expression status were remarkably correlated with OS in GC patients. In addition, results of multiple-factor analysis indicated that PIWIL1 was an independent predictor of prognosis in GC patients. The results are summarized in Table 2 .

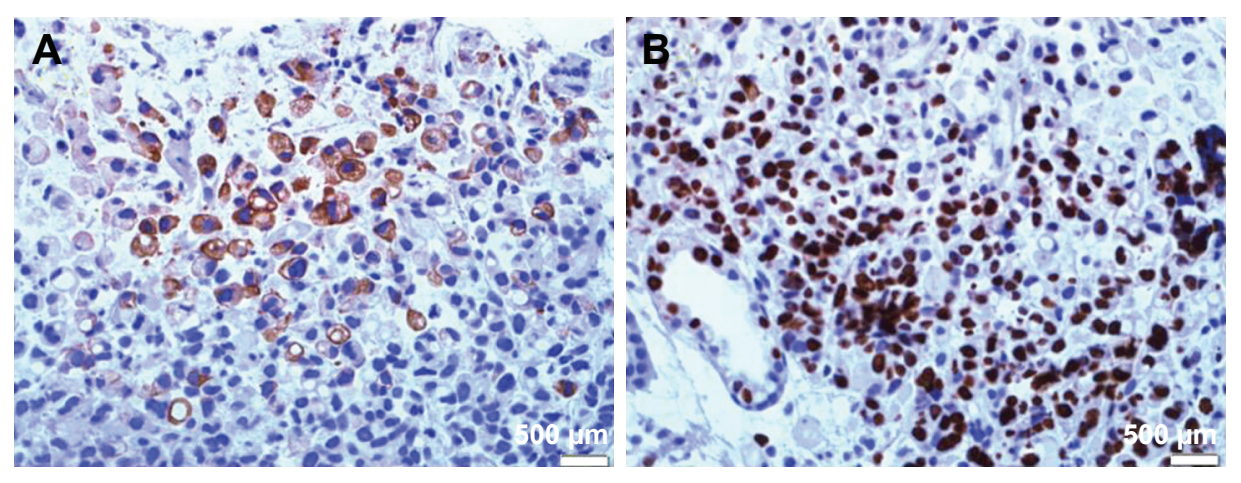

Figure I The PIWILI protein detected in cytoplasm (A) and in nucleus $(\mathbf{B})$ of the GC cells by immunohistochemical staining. Abbreviations: PIWILI, PIWI-like protein I; GC, gastric cancer. 
Table I Associations between high PIWILI expression and clinicopathological characteristics in GC patients

\begin{tabular}{|c|c|c|c|c|c|}
\hline \multirow[t]{2}{*}{ Characteristic } & \multirow[t]{2}{*}{ Patients (n) } & \multicolumn{2}{|c|}{ PIWILI expression, $\mathbf{n}(\%)$} & \multirow[t]{2}{*}{ Pearson $\chi^{2}$} & \multirow[t]{2}{*}{$P$-value } \\
\hline & & Low & High & & \\
\hline Total & 120 & 40 & 80 & & \\
\hline Gender & & & & 0.313 & 0.576 \\
\hline Male & 83 & 29 (34.9\%) & $54(65.1 \%)$ & & \\
\hline Female & 37 & II (29.7\%) & $26(70.3 \%)$ & & \\
\hline Age & & & & 0.840 & 0.359 \\
\hline$<60$ & 70 & $21(30.0 \%)$ & 49 (70.0\%) & & \\
\hline$\geq 60$ & 50 & 19 (38.0\%) & $31(62.0 \%)$ & & \\
\hline Histological type & & & & 3.676 & 0.452 \\
\hline Tubular & 104 & 35 (33.7\%) & $69(66.3 \%)$ & & \\
\hline Mixed (tubular and mucinous) & I & $0(0.0 \%)$ & $\mathrm{I}(100.0 \%)$ & & \\
\hline Mucinous & 7 & $2(28.6 \%)$ & $5(71.4 \%)$ & & \\
\hline Signet ring cell & 5 & $3(60.0 \%)$ & $2(40.0 \%)$ & & \\
\hline Others & 3 & $0(0.0 \%)$ & $3(100.0 \%)$ & & \\
\hline Differentiation & & & & 9.246 & 0.026 \\
\hline Well & 14 & $9(64.3 \%)$ & $5(35.7 \%)$ & & \\
\hline Moderate & 30 & $12(40.0 \%)$ & $18(60.0 \%)$ & & \\
\hline Poor & 63 & $15(23.8 \%)$ & $48(76.2 \%)$ & & \\
\hline Others & 13 & $4(30.8 \%)$ & $9(69.2 \%)$ & & \\
\hline Depth of invasion & & & & 6.691 & 0.010 \\
\hline Tis & 4 & $2(50.0 \%)$ & $2(50.0 \%)$ & & \\
\hline $\mathrm{Tla}+\mathrm{Tlb}+\mathrm{T} 2$ & 36 & $18(50.0 \%)$ & $18(50.0 \%)$ & & \\
\hline $\mathrm{T} 3+\mathrm{T} 4 \mathrm{a}+\mathrm{T} 4 \mathrm{~b}$ & 80 & $20(25.0 \%)$ & $60(75.0 \%)$ & & \\
\hline Lymph node metastases & & & & 6.951 & 0.008 \\
\hline No & 38 & 19 (50.0\%) & $19(50.0 \%)$ & & \\
\hline NI & 82 & $21(25.6 \%)$ & $6 \mathrm{I}(74.4 \%)$ & & \\
\hline TNM stage & & & & 8.366 & 0.004 \\
\hline 0 & 3 & $2(66.7 \%)$ & I (33.3\%) & & \\
\hline I & 22 & $12(54.5 \%)$ & $10(45.5 \%)$ & & \\
\hline II & 41 & 14 (34.1\%) & 27 (65.9\%) & & \\
\hline III & 43 & $10(23.3 \%)$ & $33(76.7 \%)$ & & \\
\hline IV & 11 & $2(18.2 \%)$ & $9(81.8 \%)$ & & \\
\hline Helicobacter pylori infection & & & & 4.156 & 0.041 \\
\hline Positive & 99 & $29(29.3 \%)$ & 70 (70.7\%) & & \\
\hline Negative & 21 & II (52.4\%) & $10(47.6 \%)$ & & \\
\hline
\end{tabular}

Abbreviations: PIWILI, PIWI-like protein I; GC, gastric cancer; Tis, carcinoma in situ.

\section{In vitro cell experiment}

PIWIL1 expression levels in GC cell lines (NCI-N87, AGS, and MGC803) and a normal gastric epithelial cell line (GES-1) were detected through Western blotting analysis. PIWIL1 expression levels in tumor cell lines were higher than in the normal gastric epithelial cell line, and were highest in the GC cell line MGC803 (Figure 3). Therefore, the MGC803 cell line was used for subsequent experiments.

To explore the relationship between PIWIL1 and the GC cell phenotype, we knocked down PIWIL1 expression in MGC803 cells through siRNA, and the siRNA transfection efficiency was verified by Western blotting analysis (Figure 4A). As shown by CCK-8 detection, PIWIL1-knockdown cells showed slower growth than those in the control group (Figure 4B). In the Transwell assay, compared with the control group, PIWIL1-knockdown cells had markedly reduced invasion and migration capacities (Figure 4C). Furthermore, they had lower wound-healing capacity as measured by the scratch assay (Figure 4D). Collectively, these results suggested that PIWIL1 knockdown could suppress the proliferation, invasion, and migration of MGC803 cells.

\section{Discussion}

PIWI regulates gene expression mainly through specific binding with PIWI-interacting RNAs, which are involved in a variety of biological processes. ${ }^{4,6}$ Its precise regulatory mechanisms include gene silencing, transposon silencing, translation inhibition, and epigenetic changes. ${ }^{9}, 10$ PIWIL1 is located on 12q24.33 and encodes an 861-residue polypeptide that is $98.6 \mathrm{kDa}$ in size. ${ }^{11}$ PIWIL1 is extensively expressed 
A

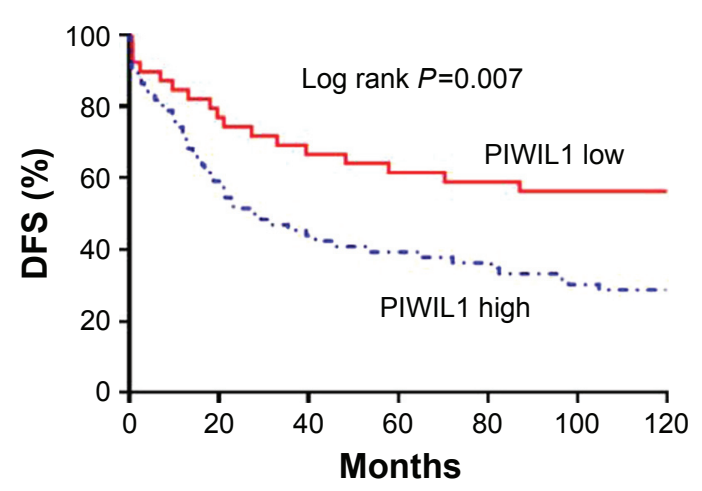

C

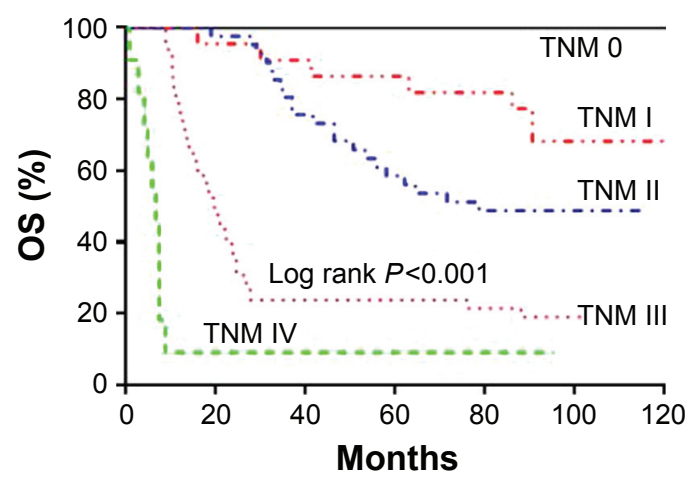

B

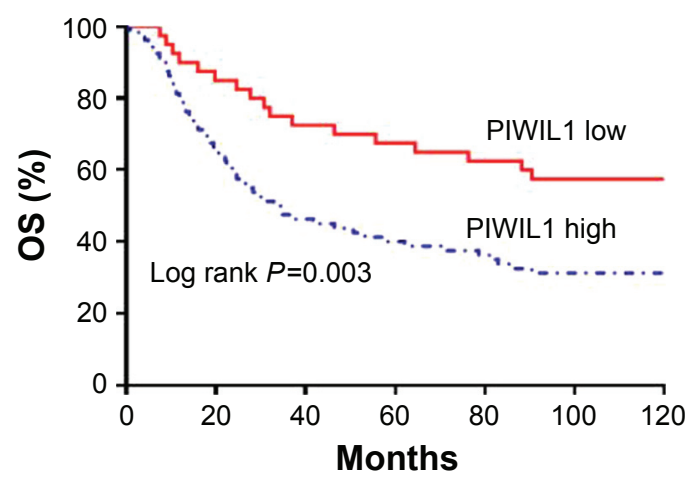

D

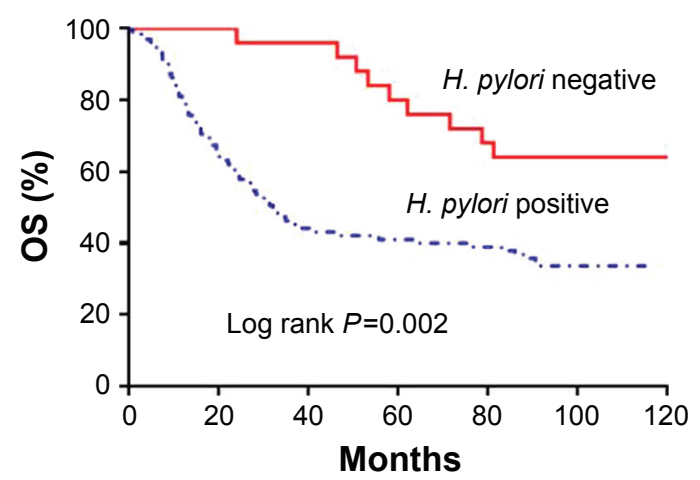

Figure 2 (A) Kaplan-Meier analysis of DFS for GC patients according to the PIWILI expression status. (B) Kaplan-Meier analysis of OS for GC patients according to the PIWILI expression status. (C) Kaplan-Meier analysis of OS for GC patients according to the TNM stage. (D) Kaplan-Meier analysis of OS for GC patients according to H. pylori infection.

Abbreviations: DFS, disease-free survival; OS, overall survival; H. pylori, Helicobacter pylori; PIWILI, PIWI-like protein I; GC, gastric cancer.

Table 2 Univariate and multivariate analysis of prognostic markers for OS in GC patients

\begin{tabular}{|c|c|c|c|c|c|c|}
\hline & \multicolumn{3}{|c|}{ Univariate analysis } & \multicolumn{3}{|c|}{ Multivariate analysis } \\
\hline & HR & $95 \% \mathrm{Cl}$ & $P$-value & HR & $95 \% \mathrm{CI}$ & $P$-value \\
\hline \multicolumn{7}{|l|}{ PIWILI expression } \\
\hline \multicolumn{7}{|l|}{ Gender } \\
\hline Male vs female & 1.061 & $0.643-1.753$ & 0.816 & - & - & - \\
\hline $\begin{array}{l}\text { Age } \\
\qquad<60 \text { vs } \geq 60\end{array}$ & 0.959 & $0.600-1.532$ & 0.861 & - & - & - \\
\hline \multicolumn{7}{|l|}{ Histological type } \\
\hline $\begin{array}{l}\text { Tubular vs mixed (tubular and mucinous) } \\
\text { vs mucinous vs signet ring cells vs others }\end{array}$ & 0.982 & $0.773-1.247$ & 0.882 & - & - & - \\
\hline \multicolumn{7}{|l|}{ Differentiation } \\
\hline Well vs moderate vs poor & 1.293 & $0.967-1.729$ & 0.083 & - & - & - \\
\hline Depth of invasion & & & & & & \\
\hline Tis vs $\mathrm{TI}$ vs $\mathrm{T} 2$ vs $\mathrm{T} 3$ vs $\mathrm{T} 4$ & 1.946 & $1.198-3.160$ & 0.007 & - & - & - \\
\hline Lymph node metastases & & & & & & \\
\hline No vs NI vs N2 vs N3 & 2.424 & $1.431-4.105$ & 0.001 & - & - & - \\
\hline TNM stage & & & & & & \\
\hline 0 vs I vs II vs III vs IV & 3.121 & $2.319-4.202$ & $<0.001$ & 3.031 & $2.303-4.533$ & $<0.00$ I \\
\hline Helicobacter pylori infection & & & & & & \\
\hline Positive vs negative & 2.890 & I.434-5.823 & 0.003 & $2.37 \mid$ & I.652-2.884 & 0.006 \\
\hline
\end{tabular}

Abbreviations: OS, overall survival; GC, gastric cancer; PIWILI, PIWI-like protein I; Tis, carcinoma in situ. 

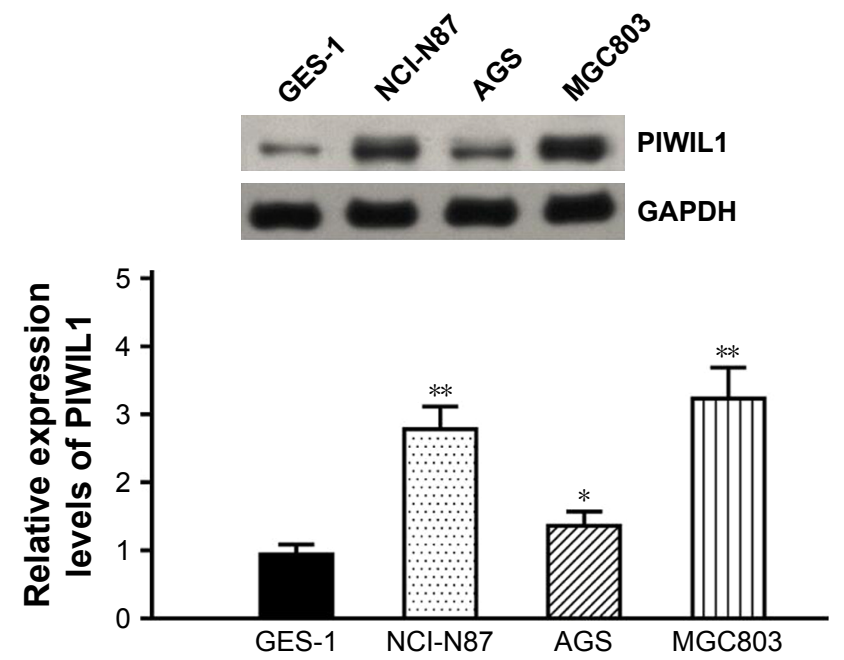

Figure 3 The PIWILI protein detected in GC cell lines and normal gastric epithelial cell line by Western blotting.

Notes: $* P>0.05 ; * * P<0.05$.

Abbreviations: PIWILI, PIWI-like protein I; GC, gastric cancer.

in human tissues, including the prostate, ovary, brain, liver, heart, kidney, and skeletal muscle. ${ }^{11,12}$ PIWIL1 plays a key role in the self-renewal of stem cells and RNA interference, which can also affect cancer cell proliferation. Moreover, PIWIL1 is closely related to the biological behaviors of tumor cells such as proliferation, apoptosis, adhesion, migration, and chemoresistance. ${ }^{13,14}$ Wang et al investigated the expression of the human PIWI subfamily proteins (PIWIL1-4) in GC and their potential roles in the occurrence, development, and prognosis of GC. They found that proteins of the PIWI subfamily (including PIWI-1) are upregulated in GC tissues and are associated with prognosis in patients with GC. ${ }^{15}$ However, independent research on the relationship between PIWIL1 and GC has not been reported, especially in in vitro cell experiments.

In this study, we found that PIWIL1 is highly expressed in GC tissues. We analyzed clinical data from 120 GC patients, and found that PIWIL1 expression status was closely associated with the tumor differentiation degree, tumor size, and TNM stage. All GC patients in this study had extensive follow-up, and we found that GC patients with high PIWIL1 expression had decreased survival. Moreover, multiple-factor regression analysis suggested that high PIWIL1 expression is an important marker that independently predicts dismal prognosis for GC patients. The abovementioned research results suggest that PIWIL1 may promote the growth, proliferation, and invasion of GC cells. However, these are retrospective
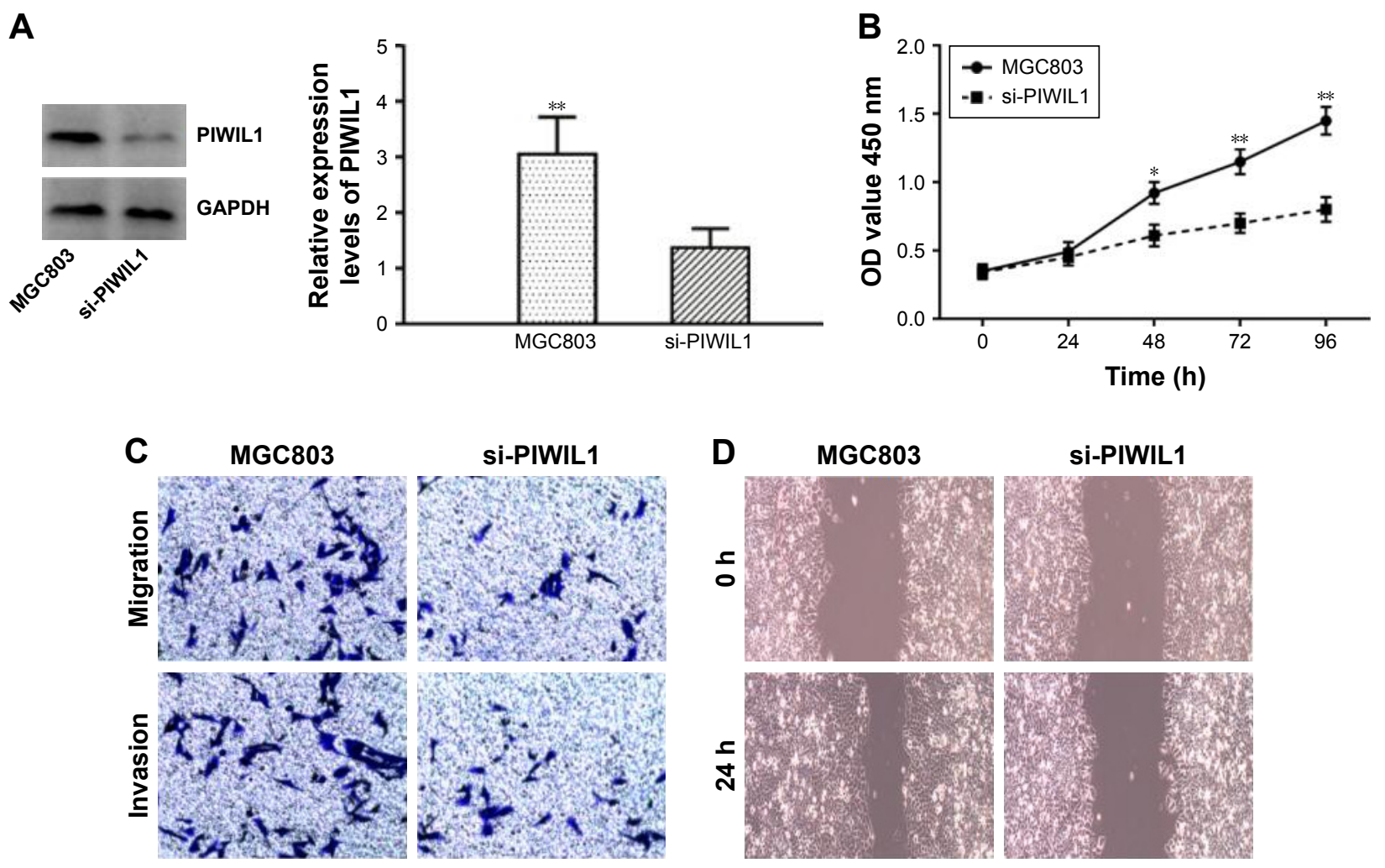

si-PIWIL1

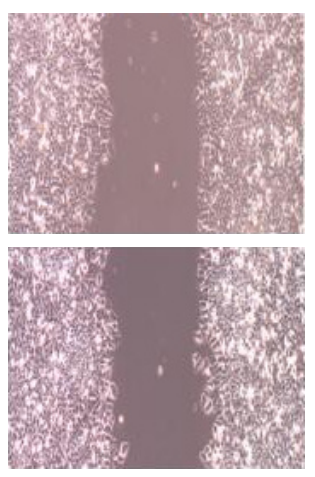

Figure 4 (A) Results of Western blotting showed that PIWILI-targeting siRNA downregulated its expression in MGC803 cells. (B) Proliferation of PIWILI-silenced MGC803 cells was measured by the CCK-8 assay. (C) The migration and invasion of MGC803 cells after PIWILI-targeting siRNA transfection as determined by the Transwell assay. (D) The migration of MGC803 cells after PIWILI-targeting siRNA transfection as determined by the wound-healing assay. Notes: $* P<0.05 ; * * P<0.01$. 
patient results that require further verification in vitro. Consequently, we silenced PIWIL1 expression in MGC803 cells and found that downregulation of PIWIL1 expression can suppress the proliferation, invasion, and migration of GC cells. Furthermore, we found that PIWIL1 may affect the malignant phenotype of GC cells. In a lung cancer model, PIWIL1 is activated by Ras-association domain family $1 \mathrm{C}$ protein. ${ }^{16,17}$ Ras-association domain family $1 \mathrm{C}$ protein can induce ERK1/2 phosphorylation in cancer cells, thereby activating the MEK-ERK1/2 signaling pathway to upregulate PIWIL1 expression. ${ }^{18}$ This results in the infinite self-renewal of tumor stem cells and thereby promotes tumorigenesis and development.

\section{Conclusion}

High PIWIL1 expression reveals dismal prognosis for GC patients, and PIWIL1 can serve as an important molecular marker for prognosis in GC patients. However, the precise role of PIWIL1 in gastric tumorigenesis remains unclear and requires further investigation. We believe that blocking PIWIL1-related signal transduction can delay tumor proliferation, invasion, and migration.

\section{Acknowledgment}

This work was supported by the Research Development Fund of the Department of Oncology.

\section{Disclosure}

The authors report no conflicts of interest in this work.

\section{References}

1. Ferlay J, Soerjomataram I, Dikshit R, et al. Cancer incidence and mortality worldwide: sources, methods and major patterns in GLOBOCAN 2012. Int J Cancer. 2015;136(5):E359-E386.

2. Schwarz RE. Current status of management of malignant disease: current management of gastric cancer. J Gastrointest Surg. 2015;19(4):782-788.
3. Frank NY, Schatton T, Frank MH. The therapeutic promise of the cancer stem cell concept. J Clin Invest. 2010;120(1):41-50.

4. Juliano CE, Reich A, Liu N, et al. PIWI proteins and PIWI-interacting RNAs function in Hydra somatic stem cells. Proc Natl Acad Sci U SA. 2014;111(1):337-342.

5. Liu WK, Jiang XY, Zhang ZX. Expression of PSCA, PIWIL1, and TBX2 in endometrial adenocarcinoma. Onkologie. 2010;33(5):241-245.

6. Zeng L, Zhang Q, Yan K, Zhou MM. Structural insights into piRNA recognition by the human PIWI-like $1 \mathrm{PAZ}$ domain. Proteins. 2011;79(6):2004-2009.

7. Sun R, Gao CL, Li DH, Li BJ, Ding YH. Expression Status of PIWIL1 as a Prognostic Marker of Colorectal Cancer. Dis Markers. 2017;2017: 1204937-1204947.

8. Graziosi L, Marino E, Cavazzoni E, Donini A. Prognostic value of the seventh AJCC/UICC TNM classification of non-cardia gastric cancer. World J Surg Oncol. 2013;11:103.

9. Gebert D, Rosenkranz D. RNA-based regulation of transposon expression. Wiley Interdiscip Rev RNA. 2015;6(6):687-708.

10. Sugimoto K, Kage H, Aki N, et al. The induction of H3K9 methylation by PIWIL4 at the p16Ink4a locus. Biochem Biophys Res Commun. 2007;359(3):497-502.

11. Girard A, Sachidanandam R, Hannon GJ, Carmell MA. A germlinespecific class of small RNAs binds mammalian Piwi proteins. Nature. 2006;442(7099):199-202.

12. Kwon C, Tak H, Rho M, et al. Detection of PIWI and piRNAs in the mitochondria of mammalian cancer cells. Biochem Biophys Res Commun. 2014;446(1):218-223.

13. Xie K, Zhang K, Kong J, et al. Cancer-testis gene PIWIL1 promotes cell proliferation, migration, and invasion in lung adenocarcinoma. Cancer Med. 2018;7(1):157-166.

14. Wang X, Sun S, Tong X, et al. MiRNA-154-5p inhibits cell proliferation and metastasis by targeting PIWIL1 in glioblastoma. Brain Res. 2017; 1676:69-76.

15. Wang Y, Liu Y, Shen X, et al. The PIWI protein acts as a predictive marker for human gastric cancer. Int J Clin Exp Pathol. 2012;5(4): 315-325.

16. Reeves ME, Firek M, Jliedi A, Amaar YG. Identification and characterization of RASSF1C piRNA target genes in lung cancer cells. Oncotarget. 2017;8(21):34268-34282.

17. Reeves ME, Firek M, Chen ST, Amaar YG. Evidence that RASSF1C stimulation of lung cancer cell proliferation depends on IGFBP-5 and PIWIL1 expression levels. PLoS One. 2014;9(7):e101679.

18. Reeves ME, Baldwin ML, Aragon R, et al. RASSF1C modulates the expression of a stem cell renewal gene, PIWIL1. BMC Res Notes. 2012;5:239
OncoTargets and Therapy

\section{Publish your work in this journal}

OncoTargets and Therapy is an international, peer-reviewed, open access journal focusing on the pathological basis of all cancers, potential targets for therapy and treatment protocols employed to improve the management of cancer patients. The journal also focuses on the impact of management programs and new therapeutic agents and protocols on

\section{Dovepress}

patient perspectives such as quality of life, adherence and satisfaction. The manuscript management system is completely online and includes a very quick and fair peer-review system, which is all easy to use. Visit http://www.dovepress.com/testimonials.php to read real quotes from published authors. 